\title{
Utility of Airborne Lidar Bathymetry in Extreme Coastal Environments: Planning Considerations, Results and Lessons Learned
}

Don VENTURA, United States

Fugro Pelagos Incorporated

Topic: A: Innovations in LIDAR Data Acquisition Techniques

\section{INTRODUCTION}

Over the past two years, Fugro Pelagos has undertaken Airborne Lidar Bathymetry (ALB) surveys in two particularly diverse environments: the high temperature, tropical, complex coralline Red Sea coastline of the Kingdom of Saudi Arabia; and the near-freezing, Arctic summer conditions found in the Northern Territories of Canada. These surveys, conducted on behalf of the General Commission for Surveys of Saudi Arabia (GCS) and the Canadian Hydrographic Service (CHS) respectively, were required to attain very similar accuracies and degrees of coverage to support national nautical charting requirements to international standards.

Although the outline requirements from the two agencies were similar in many respects, the polarity of the respective survey area environmental conditions necessitated a completely different response to the factors affecting successful conduct of the projects. Airborne operations can be affected by a plethora of issues, ranging from platform support, logistics and maintenance; climatological and oceanographic trends and events; through to ALB system and personnel performance and workload under differing operational pressures.

This paper will describe the projects in outline, the requirements of the surveys, system and aircraft parameters, and the different issues determined for both survey environments, together with the solutions to these issues. The paper will aspire to compare and contrast the various factors that need to be taken into consideration when operating ALB in such diverse environments whilst still trying to attain the same final product accuracies and standards.

\section{OUTLINES OF THE SURVEY AREAS}

\section{Saudi Arabian Red Sea Surveys 2010}

In 2009, a hydrographic charting project The Red Sea Coastal Zone, Area between "Sha'ab Naza" and "Sha'ab Al Kaber" was generated by Saudi Arabia's General Commission for Survey (GCS) and opened for tender for an IHO S44 Order 1a \& 2 survey suitable for ENC/charting use. A focus of the programme was to assess the level of effective hydrographic data acquisition in a typically diverse Saudi marine area, not least the dynamic requirements of the Kingdom's main Red Sea port at Jeddah. A Fugro bid won the contract based on a comprehensive combined LiDAR/MBES programme.
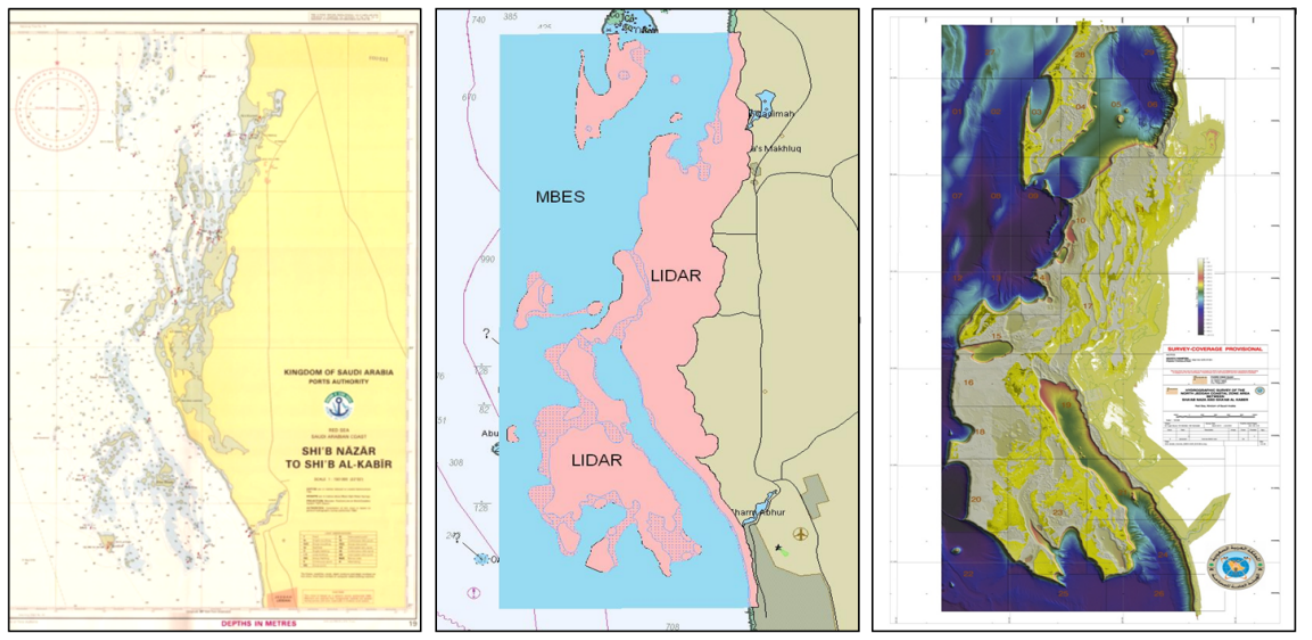

Figure 1: North Jeddah combined MBES/LiDAR survey area. (From left) Original Saudi chart, projectdesignation between LiDAR/MBES, and final combined acquired MBES/LiDAR survey results 
Initial designated assignment of the survey polygons was established based on the operational efficiencies and limitations of the main bathymetric technologies. Data density requirements, particularly for LiDAR, were pre-calculated in order to achieve the appropriate seabed coverage and target detection criteria specified in the associated contract specifications. This generated a data density based on a $3 \mathrm{~m} \times 3 \mathrm{~m}$ spot spacing pattern conducted at a minimum of $200 \%$ coverage $(60 \%$ effective runline overlap) to create a nominal density of LiDAR data sufficient to guarantee the target detection criteria required for IHO S-44 Order 1a. LiDAR was optimized in all areas where navigation hazards and/or shallow water were considered significant to either crew and vessel safety or operational efficiency (due to reduced swath coverage) of the MBES systems. By accepting a conservative limit for LiDAR penetration but equally conservative for ship safety, a healthy data overlap was assured and this facet of operations, subject to amendment as the survey progressed for the reasons provided below, greatly aided the complex data merge operation during the final processing stages of the survey.

Subsequently, GCS issued instructions for this to be carried out and the UKHO-supervised IMO submission for the Approaches and Routes to the new King Abdullah Port were presented before the IMO in July 2010.

\section{Canadian Arctic Surveys 2011}

Fugro was contracted during August 2011 by the Canadian Hydrographic Service (CHS) to conduct an airborne bathymetric LiDAR survey in specific areas of the Canadian Arctic at various times and locations and deliver fully processed and verified hydrographic survey data. The goal of the project was to investigate the feasibility of the implementation of bathymetric LiDAR into the hydrographic survey program in Canada. The airborne LiDAR survey was conducted with the SHOALS-1000T system for data collection to the extents provided by the CHS.

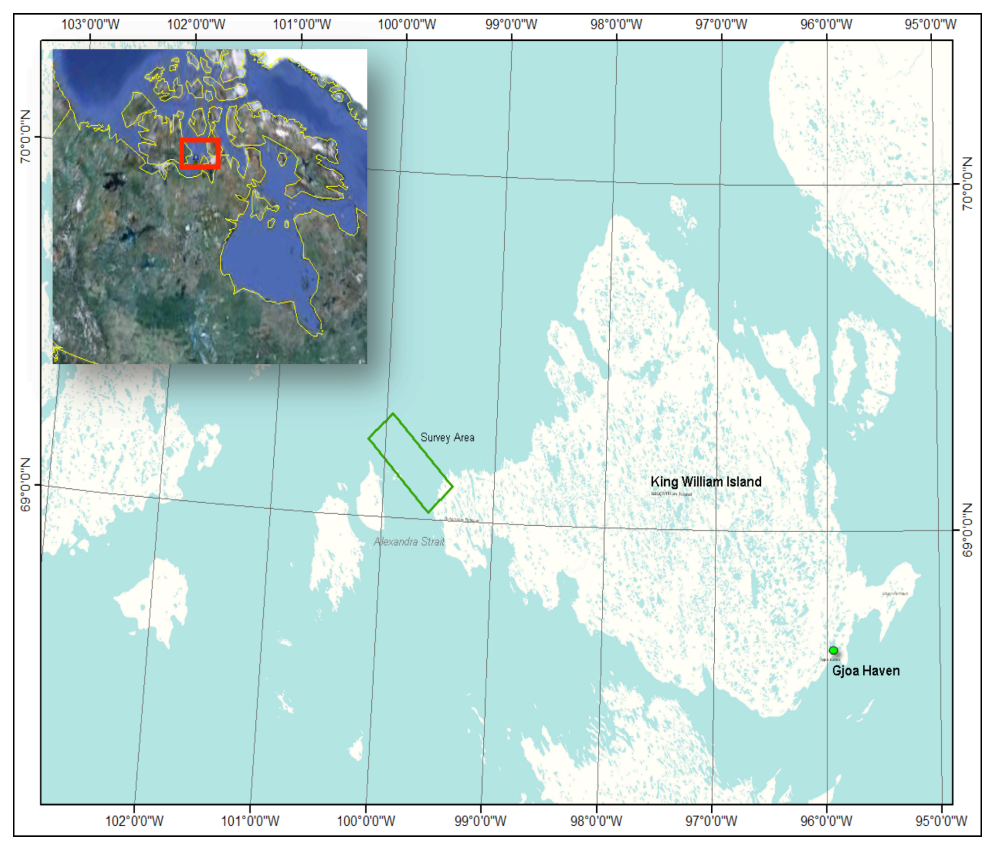

In this case, the airborne bathymetric LiDAR survey was planned to achieve IHO SP-44 Order $1 \mathrm{~b}$ category of survey coverage and accuracy. This was accomplished by combining a $5 \mathrm{~m} \times 5 \mathrm{~m}$ spot spacing (flying at $400 \mathrm{~m}$ altitude and speed-over-ground of approximately 160 knots) with a $100 \%$ coverage plan. Planned line spacing provided $30 \mathrm{~m}$ of sidelap. The reduction in point density for this survey compared to the Red Sea survey did not affect in any way the individual point accuracy criteria, with both projects meeting S-44 Order 1 horizontal and vertical accuracy standards.

Figure 2: One of the CHS LiDAR Survey Areas (Alexandra Strait)

\section{TECHNICAL AND LOGISTIC COMMONALITIES BETWEEN THE SURVEYS}

Both surveys were flown with sufficient options, made available to the airborne operator, to devise a best 'plan of the day' for climatic and water quality considerations, such that successful data collection was possible in both shallow and deep regions of the areas under consideration. Operator assessments included reconnaissance of areas for water turbidity issues and wind direction and strength affecting survey parameters which were highly variable due to the predominant climatic factors being experienced on any given day.

The survey acquisition operations collected data from the following sources:

- $\quad$ Bathymetric LiDAR data from the SHOALS-1000T system. 


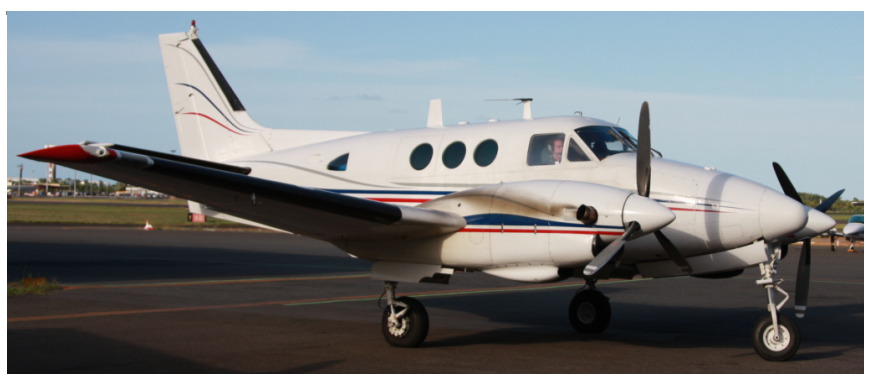

Figure 3: Beechcraft King Air A90
GPS base stations were installed in or near the vicinity of the survey areas such that the range of operations from the base stations did not exceed current standard operating procedures for this type of survey (typically $<70 \mathrm{~km}$ ). Base station data were typically collected every day from each station when a flight mission was planned. Back-up secondary stations at each location were set up for redundancy purposes.

Surveys in both regions were conducted from the same type of aircraft and indeed from the same provider; this in itself also created a standardised operational 'style' or paradigm, with the aircrew providing a uniform level of service and conduct of air operations.

\section{TECHNICAL AND LOGISTIC DIFFERENCES BETWEEN THE SURVEYS}

Whilst few if any technical differences existed between the survey approaches from a bathymetric LiDAR perspective, which shared the same types of survey equipment, sensors and even aircraft type, considerable logistic differences were evident between the projects. These included mobilization distances, local population centres providing logistic and other support services, shipping and transit considerations, accommodation, storage and victualling/refuelling arrangements. Although none of these aspects directly affected the end result of the survey or nature of the data collected, it is worthy of mention if only to recognise that these differences did exist.

\section{HANDLING EXTREMES - ENVIRONMENTAL}

As the main topic of this paper suggests, the mitigation of the primary environmental factors required for the successful prosecution of these surveys was considerable in both regions and equally necessary. The extremes of these climates highlighted the need for different approaches and planning to address a common set of issues on which successful airborne bathymetric LiDAR operations depended. These were: temperature and humidity; cloud cover and ceiling; precipitation; winds; surface waves; tidal streams and currents; and water clarity/turbidity. We will discuss these effects on bathymetric LiDAR operations in turn and compare the effects as they pertain to these two regions.

\section{Temperature and Humidity}

With respect to the typical bathymetric LiDAR sensor, high ambient air temperatures and humidity necessitate air-conditioning in an aircraft conducting such operations with today's technology, not just for personnel comfort. The aircraft cabin atmosphere thus needs to be conditioned in order to maintain Lidar system temperatures within design specifications. Low ambient air temperatures can also cause system operation parameters to be exceeded, with stability of the laser triggering mechanisms and electronics similarly affected. The mitigation is the same; to provide a stable, controlled airflow to the primary sensors to ensure consistent performance and reduce the likelihood of environmental stress on the main and most sensitive components.

From a platform standpoint, air temperature affects density, lower atmosphere turbulence and therefore operational performance of the aircraft. Thermals generated near a coastline are often very violent due to the different refractive heat indices that water and land (especially dry land) generate. Katabatic effects of descending cold air from nearby steep terrain can cause similarly unpredictable and unstable operating conditions. Severe turbulence can render an otherwise optimal data collection scenario almost impossible to conduct safely. Heat haze can also diffract the laser energy in the air column, resulting in reduced depth of penetration results compared to that expected in an otherwise clear water scenario.

In these examples, mean temperatures in the Red Sea area were lower than $30^{\circ} \mathrm{C}$ from November to March, while humidity was lower from April to November, indicating that the optimum data collection 
based on just this factor would be during the winter months. Conversely for the Canadian areas, the average temperatures throughout the year varied from $-30^{\circ} \mathrm{C}$ in January to $7^{\circ} \mathrm{C}$ in July. Only the brief summer hiatus, where temperatures are above freezing, provided conducive conditions for the efficient collection of quality LIDAR data given the low altitude flying and requirements to have an ice-free survey and safe aircraft operating environment.
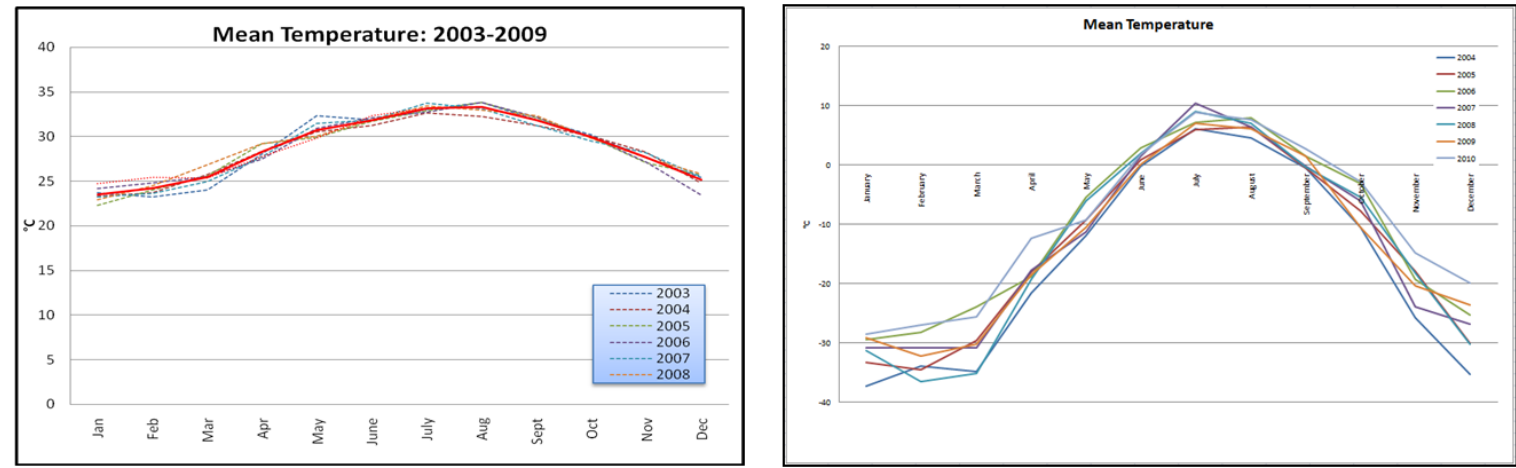

Figure 4: Mean temperatures: Red Sea (Jeddah) and Canadian Arctic (Gjoa Haven)

High humidity, irrespective of temperature, can affect the performance of the sensor, potentially causing condensation or corrosion in the equipment. Humid conditions, admittedly usually in concert with higher air temperatures, can also create a hazy environment and absorption of part of the laser energy being returned to the detectors/receivers.

\section{Cloud Cover and Ceiling}

Cloud cover, and specifically the ceiling altitude, can affect airborne hydrographic LIDAR operations. LIDAR bathymetry data collection is typically planned to be collected from an altitude of between 300 and $500 \mathrm{~m}$ or so. This means the ceiling for cloud, haze or fog must be at least this high. This aspect of the environment is seldom a major issue in the Red Sea, although other unusual phenomena affecting visibility through the air column, namely dust storms, were an issue that were harder to predict until sufficient local knowledge regarding the prevalence of high winds combined with local terrain capable of providing the particulates was understood. In the Arctic however the degree of low cloud cover was a regular phenomenon, particularly in coastal areas where advection created low cloud or fog. In circumstances like these a real risk of aborted data collection missions exists and there is very little mitigation possible unless the survey is sufficiently expansive to allow for alternative areas to be selected when the preferred area is not possible to survey.

\section{Precipitation}

Rainfall can be a problem for hydrographic Lidar operations in two ways. Firstly, there is a significant loss of laser energy as the light scatters off raindrops in the atmosphere. Secondly, localized run-off from a rain event will often negatively impact water clarity in and around the coastal margins.

As one would expect, the selected regions in this paper again highlighted the maxima and minima effects of this factor on operations. Whilst it was not a major factor in the Red Sea as expected, precipitation in the form of ice, snow and rain was experienced in the Arctic. This not only affected the sensor performance but, in extremis, affected safe air operations when heavy overnight snowfalls were encountered making use of the local airport runways more hazardous than usual. Additionally, the higher albedo effect from falling snow creates glare issues for a bathymetric laser system, especially with a highly contrasting dark surface such as rock or water surface viewed under expansive cloud cover. If predictable (such as in some tropical conditions), then the effects of precipitation can be easily mitigated with carefully timed sorties, or provision of alternative data collection sites when the intended area is unsuitable.

\section{Winds}

High winds can impact the aircraft's ability to remain reasonably level and fly straight lines. As a general rule of thumb, winds generally need to be less than $20 \mathrm{kts}(37 \mathrm{~km} / \mathrm{h})$ for an aircraft conducting bathymetric LiDAR operations to safely and effectively conduct an aerial survey. 
Such scenarios can exist in any part of the world and coastal areas, regardless of latitude, are particularly susceptible to local increased wind effects. Both examples highlighted here created operational issues due to excessive periodic winds. This is typically mitigated by a carefully chosen survey line direction to minimise the significance of the wind force vector on the platform stability and operational (air) speed, and/or timing of the airborne sorties to avoid more predictable wind events (such as the build-up of onshore winds in the tropics created by dissimilar surface heating of land and sea. The more inclement general weather patterns of higher latitudes were less predictable, but just as frequent, as the former phenomenon experienced regularly during the Red Sea surveys, so similar downtime or interruption is experienced in completely disparate regions.

\section{Surface Waves}

Winds can also cause breaking waves, which creates air pockets in the water column and may prevent the laser beam from reaching the seabed. Breaking or cresting waves could be caused by extreme winds, in which case the aircraft would probably not be flying for safety reasons anyway. If the wave is breaking at the land / water interface, this could also create turbidity from disturbed sediments in the surf zone. The increased turbidity will decrease water clarity and may prevent the laser beam from reaching the seabed. Irrespective of the region, these effects are common to the water medium and the only mitigation is to avoid such areas during survey and to have alternate, leeward shores available as substitute areas until local conditions change.

\section{Tidal Streams and Currents}

It is possible for tidal streams and wind-generated currents to affect a hydrographic Lidar collection. If the current is extreme, it can create turbidity from disturbed sediments because of the high laminar velocities. The increased turbidity will decrease water clarity and may prevent the laser beam from reaching the seabed. If the flow is tidally influenced, surveying at slack tide can mitigate the problem, as the current is reduced at that time.

These phenomena did affect both surveys, with different drivers creating the problems. In the Red Sea, tidal ranges were seldom greater than $0.6 \mathrm{~m}$, but the propensity and complexity of the steepsided reefs throughout the survey areas created barriers to the regionally induced tidal forces and had the effect of accelerating water flow through the deeper, narrow channels between the reefs. Closer inshore where higher levels of sediment were prevalent on the seabed, this created localised turbidity events which were predictable with the state of the tide and therefore reasonably comfortably mitigated. In the Arctic surveys, the stronger tidal forces and greater ranges experienced created similar patterns of turbidity but these were more regional in extent and longer in period, more closely associated with the spring and neap tidal cycles. In this latter scenario, data acquisition can be interrupted for much longer periods and in extreme conditions, no data collection may be possible at all until a major phase change in the tidal cycle occurs.

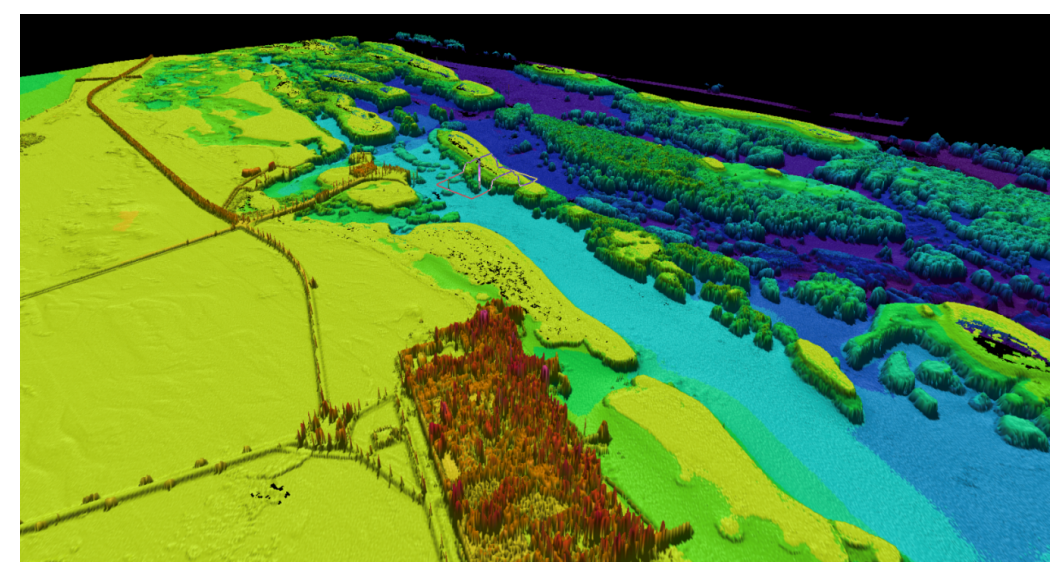

Figure 5: Complex reefs in Saudi Arabia generated accelerated local tidal streams and inshore turbidity issues

\section{Water Clarity/Turbidity}

As is very evident from above, the maximum depth of penetration on an airborne bathymetric LIDAR Survey depends on a number of factors, but ultimately the most important is the clarity of the water during acquisition. Systems in use today typically operate at the $532 \mathrm{~nm}$ wavelength and between 3 and $5 \mathrm{~mJ}$ of output energy; this provides a theoretical maximum penetration of two to three times (depending upon bottom reflectivity) that of normal human visual acuity (often expressed in terms of 
Secchi depth results or a light transmissometer (k) factor), up to a maximum depth of penetration of approximately $50 \mathrm{~m}$. The combination of output energy, diffusion of the laser 'spot', and operating altitude is designed to comply with international human eye safety regulations but is considered equally safe for all fauna illuminated by a pulse. All commercially available airborne hydrographic LIDAR systems will achieve approximately the same maximum penetration in the same waters during the same survey period.

Many of the factors above, in isolation or combined, can lead to localized or area-wide turbidity issues, and so the amalgam of all these factors must be considered carefully. Additionally, biologic and subsurface events can also create turbid conditions in the water; these include algae blooms, major biomass concentrations (especially during a spawning event), freshwater or other dissimilar liquid seeps from the seabed etc. Industrial activity and waste and other human activity such as fishing and dredging operations must also be factored into the assessments if such activities are known to occur during otherwise optimal conditions. In the examples selected, only the natural phenomena - climatic and biologic - affected turbidity in the Arctic regions, but major dredging operations in one area of the Red Sea survey seriously hampered effective data collection in a specific coastal strand and effective management of the options on when and where to survey had to be enforced.

\section{HANDLING EXTREMES - OPERATIONAL}

One of the main operational factors which differed between the surveys, tied closely to the primary environmental parameters which have been focussed on above, was the brevity of the Arctic mission day compared to that experienced the previous year in Saudi Arabia. The airfield proximity, fuel availability and transit leads were radically different between the projects which in turn limited the operational flexibility available to the acquisition team in the Canadian Arctic. These elements blur into a logistical definition but more directly affected the style and tempo of field data acquisition than the other factors mentioned above once the survey was underway. Local customs and sensitivities also factored into the operational pattern to a degree; whilst this was less true for the Arctic mission, restrictions were applied to the weekly operational tempo in Saudi Arabia and no flying was conducted on holy days. Conversely, the excellent logistic support provided which allowed uninterrupted 24-hour operations for 5 days of the week more than compensated for the no-fly restriction, which actually allowed a regular maintenance routine to occur on these days and so avoided any additional down-time.

\section{CONCLUSIONS}

Irrespective of the location, airborne bathymetric LiDAR operations must be well planned in order to be well executed; in this they are therefore no different from any other type of data acquisition mission. The factors on which this success depends however, and the importance of these factors compared to other hydrographic survey techniques, are radically different. A great many historic sources of environmental information are available to make the initial determinations on which a robust survey plan depend, but ultimately the awareness and flexibility of the surveyors in the field is essential to maximize the opportunities to acquire god quality data. The medium of light rather than sound as an energy source has both advantages and disadvantages, but successful use of the former demands an understanding of the various environmental factors on which we have focussed in this paper.

\section{BIOGRAPHY}

Don VENTURA is a hydrographic surveyor with Fugro Pelagos Inc. of San Diego, CA. A 22-year Royal Naval officer veteran, he has been involved in hydrography for over 28 years and has planned, conducted and assessed hydrographic, oceanographic and geophysical surveys throughout four main ocean basins. 